\title{
Biohydrogen and bio/mimetic solar energy conversion
}

\author{
Yutaka Amao' ${ }^{1}$ Jun Miyake ${ }^{2}$
}

Published online: 2 September 2016

(C) Springer Science+Business Media Dordrecht 2016

This special issue of "Biohydrogen and Bio/Mimetic Solar Energy Conversion" in Research on Chemical Intermediates highlights recent advances of our understanding on biohydrogen production and bio/mimetic solar energy conversion based on the natural and the artificial photosynthesis for the development of a hydrogen energy society in the near future. This issue contains ten invited and selected papers on biohydrogen and biomimetic solar energy conversion research, presented at the international conference "Asia Biohydrogen and Bioenergy 2013 and Intentional Conference on Bio/Mimetic Solar Energy Conversion 2013", that was held in Osaka, Japan, during 22-24th, November, 2013 with the Asia Biohydrogen Link and Research Center for Artificial Photosynthesis (ReCAP) of Osaka City University. There were 143 participants from more than ten countries. In addition, we had four plenary, six keynote, 26 invited, and 22 general speakers and 30 posters. We also had 11 student speakers in a student-organized session. The purpose of this conference was to provide a forum where researchers from various fields related to biohydrogen and bio/mimetic solar energy conversion came together and exchanged their useful ideas. Since this conference, we have seen much need for publishing a special issue regarding to Biohydrogen and Bio/Mimetic Solar Energy Conversion research (Photos 1, 2, 3).

Yutaka Amao

amao@ocarina.osaka-cu.ac.jp

Jun Miyake

jun_miyake@bpe.es.osaka-u.ac.jp

1 The Osaka City University Advanced Research Institute for Natural Science and Technology (OCARINA), Sugimoto, Sumiyoshi-ku, Osaka 558-8585, Japan

2 Graduate School of Engineering Science, Osaka University, Machikaneyama, Toyonaka, Osaka 560-8531, Japan 

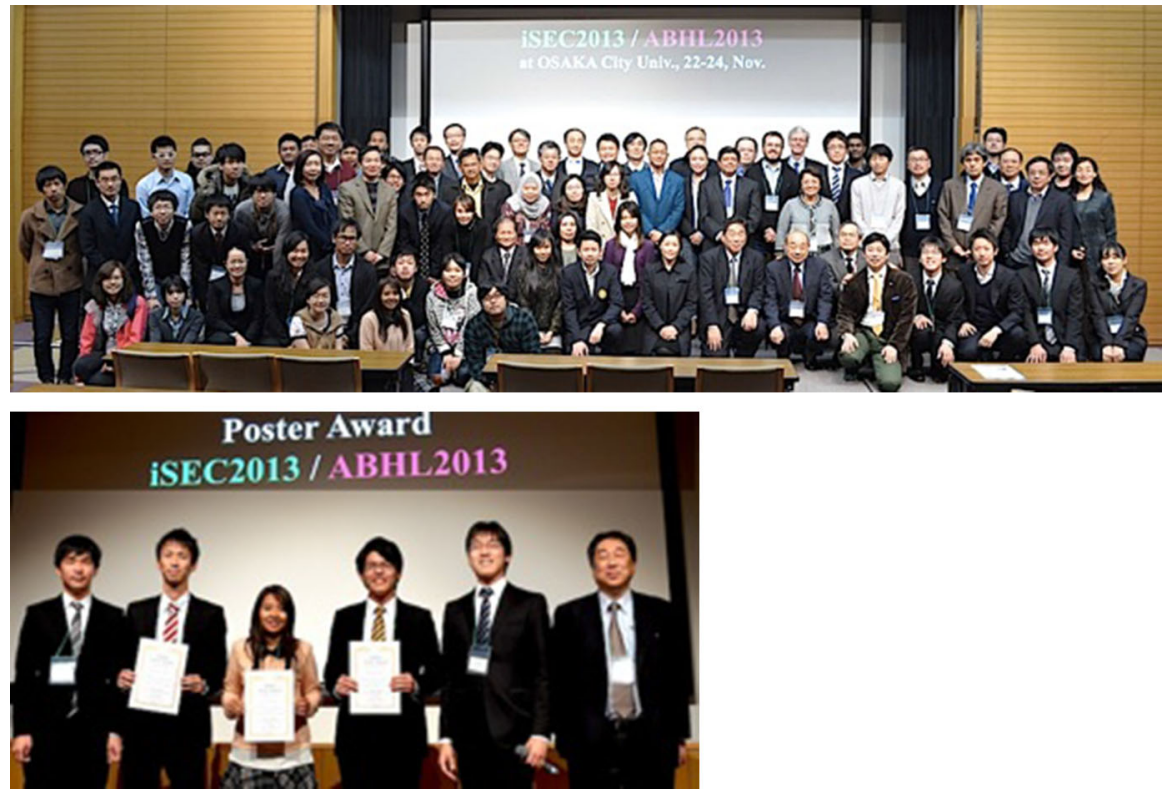

Photo 1 A group photograph on the iSEC 2013/ABHL 2013
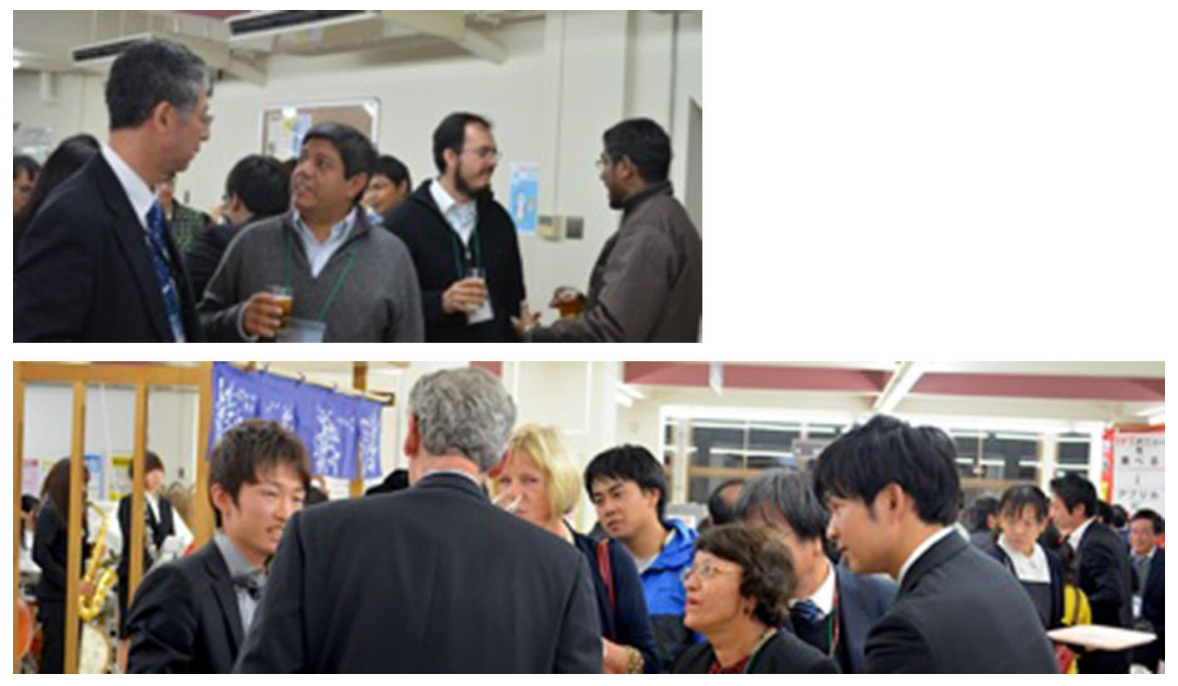

Photo 2 Some participants at the banquet 
Finally, the review of several articles was behind schedule; therefore, the publication of this special issue of "Biohydrogen and Bio/Mimetic Solar Energy Conversion" was delayed.

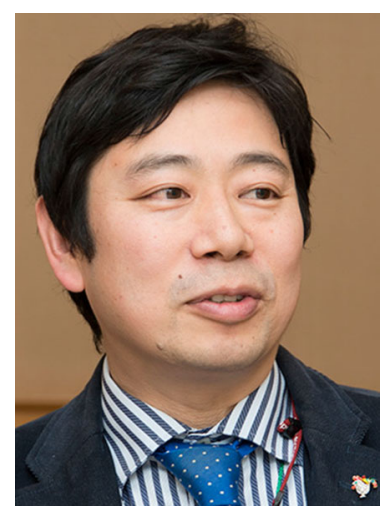

\section{Yutaka Amao}

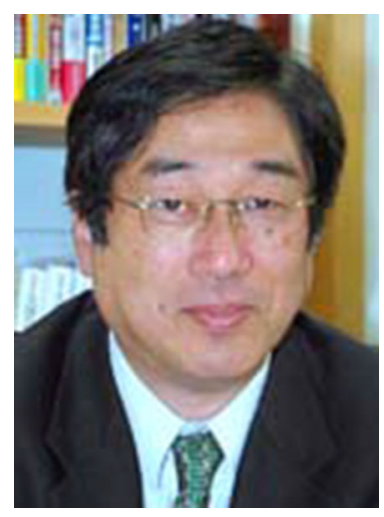

Jun Miyake

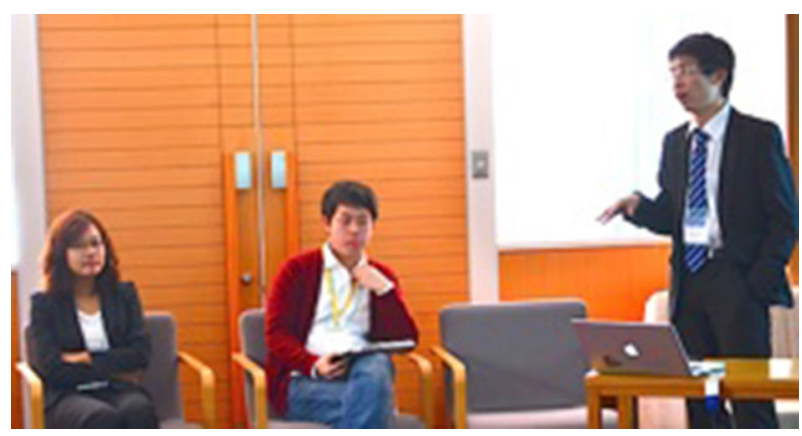

Photo 3 Student session on iSEC 2013/ABHL 2013 